\title{
Research on Energy Internet Management Mechanism from the Perspective of Cooperative Game
}

\author{
Shuang Qiao ${ }^{1}$, PengShu Wang ${ }^{1 *}$ and Pan Wang ${ }^{1}$ \\ ${ }^{1}$ School of management, dalian university of technology, dalian city, liaoning province, 116000, China
}

\begin{abstract}
Energy is closely related to the overall progress of society and the overall development of mankind. The two major problems of energy shortage and environmental pollution caused by long-term energy use have been plagued by scholars at home and abroad. The proposal of the Energy Internet aims to improve energy utilization efficiency, reduce energy waste, optimize energy allocation, and thereby alleviate environmental pollution. Therefore, the research on energy internet management is of great significance. Starting from the cooperative game theory, this paper combines the Shapley value with energy Internet management, and establishes a model to solve the problem of the distribution of excess profits caused by the joint work of the Energy Internet Alliance, and provides a new research perspective for energy Internet management.
\end{abstract}

\section{Introduction}

With the outbreak of the novel coronavirus in 2020, the global economy has been hit hard, and it is the first task to get the economy back on track after the epidemic. The Energy Production and Consumption Revolution Strategy (2016-2030) jointly issued by the National Development and Reform Commission and the National Energy Administration in 2017 proposed that by 2050, China's energy production link non-fossil energy will account for more than $50 \%$ of primary energy. Energy is the material basis for social development. The global economic development speed continues to increase, the process of industrialization will advance further, and the demand for energy will rise sharply. Through the data of the International Energy Agency, we find that by 2035, the demand for electricity in the countries of the world will increase by about $70 \%$.

The proposal of the Energy Internet highly intersects energy and Internet technologies, which can efficiently dispatch multiple energy systems and weave into an energy service network closely linked to both sides of energy supply and demand.From the perspective of cooperative game technology, it is analyzed that energy Internet management can improve the efficiency of production, transmission, consumption and other links, and realize the reasonable dispatch of energy through full use of information, thereby improving the economic benefits of each link.

\section{The characteristics and importance of energy internet management}

The Fifth Plenary Session of the 19th Central Committee emphasized that one of the key goals of high-quality economic development during the 14th Five-Year Plan period is to achieve new progress in ecological civilization, greatly increase the utilization rate of energy and resources, and continue to improve the ecological environment. Energy Internet technology has the characteristics of low carbon, clean, environmental protection, energy saving and high efficiency, and safe implementation. It uses electric energy as the central link, heat, cold, gas and other energy sources as auxiliary links, and uses Internet technology to efficiently integrate the energy system and the information and communication system. It is also an open energy interconnection network that can scientifically coordinate the production, transmission, storage, and consumption of multiple energy sources.

Characteristics ${ }^{[1]}$ of Energy Internet First, distributed characteristics. The use of multiple energy sources generally has the characteristics of being decentralized, while traditional energy sources are often unable to control the dispersed energy when they are used, which ultimately leads to the difficulty of concentration in the collection and utilization of energy. Energy Internet can collect and control the use of multiple energy sources with the help of network nodes in computer technology, thereby improving the comprehensive utilization rate of energy. Second, the characteristics of openness. In the past, the power grid could not help users understand the usage of power resources when they were in use, so to a

*Corresponding author's e-mail: 412746101@qq.com 
certain extent, it caused problems for users' electricity consumption. Energy Internet can use computer intelligent technology to share and manage energy, which improves the comprehensive utilization rate of multiple resources and can help users understand their own resource usage. Third, the characteristics of safety. In the past, energy sources encountered many hidden dangers in the process of using them, such as illegal intrusion of viruses and hacker attacks, which caused troubles to the safe operation of multi-energy sources. With the help of the Internet and multi-energy development model, the use of multi-energy can be supervised and controlled by the computer intelligent supervision and control system, and the user's personal information can be comprehensively controlled to achieve the goal of high-efficiency control of multienergy.

At present, my country's development has entered a new normal. From the relevant data released by the National Bureau of Statistics, we can obtain the proportion of China's various energy consumption in total energy consumption from 2017 to 2019 , as shown in the following figure:

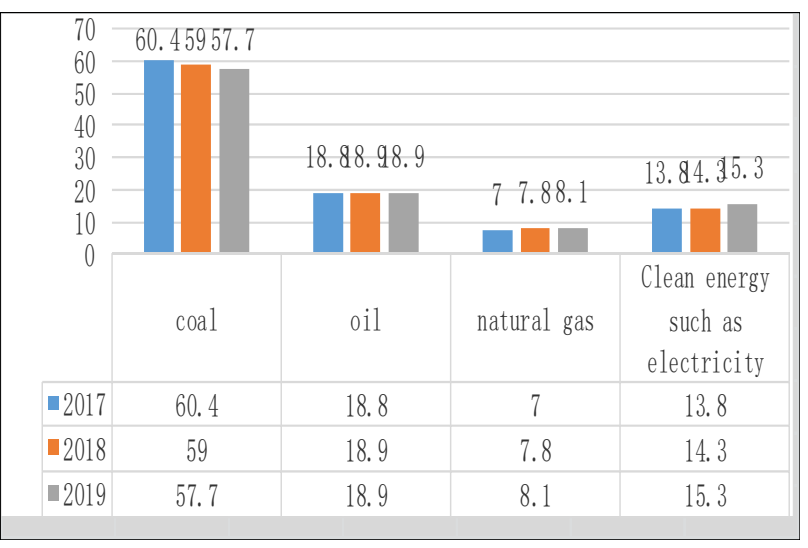

Figure1.The proportion of China's various energy consumption in total energy consumptionfrom 2017 to 2019

From the figure, we can find that the consumption of fossil en year, while the consumption of clean energy such as primary power is increasing year by year. In fact, in order to improve the efficiency of energy use, the National Energy Administration jointly issued a guideline on strengthening the standardization of the Energy Internet (hereinafter collectively referred to as the opinions) on the UN Standards Committee in 2019. The opinions emphasize the full implementation of the energy strategic thinking of "Four Revolutions, One Cooperation" under the guidance of Xi Jinping Thought on Socialism with Chinese Characteristics for a New Era. It is required to speed up the implementation of the energy Internet standardization tasks, and clearly state the overall requirements, key tasks and guarantee work. It is required to achieve a multi-industry, multi-field, energy Internet standardization system that meets the needs of my country's social development, promote the development of energy Internet technology, and achieve a new pattern of mutual development of energy, Internet and other related industries.
In addition, my country's energy structure is rich in coal, poor in oil, and low in gas. In 2019, the foreign dependence on oil and natural gas is $72 \%$ and $43 \%$, respectively, and energy security is facing severe challenges. In 2020, the utilization rate of new energy in the operation area of State Grid will reach 97.1\%. During the "14th Five-Year Plan" period, new energy will be further developed on a large scale, and the situation of efficient use of new energy will be severe. Relevant policies to lower domestic refined oil prices have promoted the development process of reducing energy costs.

The blowout development of network technology in the 21st century provides new opportunities for energy network management. One of the important ways to alleviate the environmental crisis is to access clean energy on a large scale $1^{[2]}$. Through the development of multi-field cooperation between regions, an alliance is formed. Each member of the alliance can conduct energy cooperation with other regions of the alliance according to their own needs ${ }^{[3]}$. However, multi-domain energy cooperation is related to the economic benefits of various regions and power grids. Therefore, research on reasonable income distribution when reaching a multiparticipant cooperative alliance is of great significance to the management of the energy Internet. From the perspective of cooperative game, this paper solves the problem of alliance income distribution through the combination of Shapley value and energy Internet, and provides a new research perspective for my country's energy Internet management.

\section{Establishment of income distribution model}

Since the 1940s, many scholars have begun to study how to distribute the extra wealth generated by the alliance in a fair and reasonable manner. In 1944, foreign scholars VonNeumann and Morgenstern co-authored a book called "Theory of game and economic behavior". The theoretical framework of game theory was presented for the first time, and the concept of cooperative game was also mentioned in the work ${ }^{[4]}$. In 1953, Gillies proposed the concept of "nucleus" as a tool for studying stable sets, providing a new theoretical method for studying economic markets. In the same year, Shapley wrote three axioms based on the principle of fair distribution of income and proposed a simple and analytical expression to calculate economic benefits. The advantage is that, regardless of whether the core solution is empty or not, the Shapley value can be easily calculated by the formula ${ }^{[5]}$. In 1969, Schmeidler proposed to measure the attitude of the alliance by exceeding the value, and minimize the dissatisfaction of the alliance as the solution of the cooperative game-Nucleolus. From 1964 to 1979 , Aumann and Maschler et al. proposed a series of solutions to cooperative games, such as stable solutions, Strong Nash Equilibrium, Coalition Structure Core, and Bargaining Set.

However, the above mentioned equilibrium concepts are static. In order to overcome this weakness, Chwe 
proposed the largest consistent set (Largest Consistent Set) as the solution of the cooperative game in 1994. In 1997, Owen proposed the Owen value, and based on the Shapley value, it considered the impact of the allocation of costs among alliances on the alliance structure.

The research of cooperative game is that when there is an alliance composed of more than two participants, the participants in this alliance can cooperate or act together to increase the economic benefits of the participants. Therefore, analyzing energy Internet management from the perspective of cooperation game, it is possible to more rationally distribute the alliance formed by various regions and power grids to create more economic benefits through joint cooperation. It plays a coordinated and optimized role in multi-domain energy dispatch, makes the allocation of multiple resources more reasonable, improves energy utilization efficiency, and makes overall arrangements in time and space.

The cooperative game contains two basic elements: the set of participants $\mathrm{N}$ and the characteristic function $\mathrm{T}$. Let $\mathrm{N}=\{1,2,3, \ldots, \mathrm{n}\}$ be the collection of power generation, grid dispatch, energy storage, electricity, market, service and management, and $\mathrm{n}$ represents the number of subjects participating in the cooperative game in each link. T represents the income generated by the members of the non-empty subset $\mathrm{S}$ of the participant set $\mathrm{N}$ through mutual aid. Therefore, the cooperative game can be expressed as $<\mathrm{N}, \mathrm{T}>$.For the cooperative game of transferable payment, The income distribution $\vec{X}=\left(\mathrm{X}_{1}\right.$, $\left.\mathrm{X}_{2}, \mathrm{X}_{3}, \ldots, \mathrm{X}_{\mathrm{n}}\right)$ is a payment vector. Among them, $\mathrm{X}_{\mathrm{i}}$ represents the income distributed by the alliance to member $i$. The cooperative game contains the following 6 concepts:

(1) Marginal contribution: The calculation method for the marginal contribution of participant $i$ in the Energy Internet Alliance is as follows.

$$
M C_{i}(S)=v(S)-v(S-\{i\})
$$

$\mathrm{MC}_{\mathrm{i}}(\mathrm{S})$ represents the marginal contribution of the participant $\mathrm{i}, \mathrm{v}(\mathrm{S}) \mathrm{i}$ and $\mathrm{v}(\mathrm{S}-\{\mathrm{i}\})$ respectively represent the feature function of whether the participant $i$ is in the alliance $\mathrm{S}$.

(2) Individual rationality: For utility transferable games, the payment vector should satisfy individual rationality, and each participant in the Energy Internet Alliance will get higher profits than if they were independent.

(3) The overall rationality constraint; for the transferable utility game, the payment vector also needs to satisfy the overall rationality, that is, the total income of the alliance is equivalent to the sum of the economic income distributed by the players in the game.

(4) Effective distribution: If and only if the payment vector satisfies both overall rationality and individual rationality, the payment vector is called effective distribution. Among them, individual rationality guarantees that the participating entities of the alliance obtain no less than the economic benefits of their respective independent games, and the overall rationality guarantees the reasonable distribution of the net income of the alliance within the Energy Internet Alliance.

(5) Core: The core of the transferable utility game $<\mathrm{N}, \mathrm{T}>$ represents a benefit distribution strategy that can be accepted by all participating entities in the Energy Internet Alliance. Since operating independently or forming a new alliance cannot obtain more benefits, none of the game participants has the will to operate independently from the general alliance or form a new alliance. Therefore, if the payment vector in the core needs to take into account the requirements of overall rationality and individual rationality, it must also consider alliance rationality. Alliance rationality means that the sum of the benefits obtained by the participants in any subset $\mathrm{S}$ of the energy Internet alliance participating in the main set $\mathrm{N}$ is not less than the benefits of the new alliance. However, the core set has strict premises. Therefore, not all alliances have a core, and the income distribution method in the core is usually not unique.

(6) Shapely value: The Shapley value method is used to determine the return value of the game participants in the cooperative game. The Shapley value is the only definite solution for the utility transferable alliance game. It needs to satisfy both the overall rationality and the individual rationality. The economic return of member $i$ is calculated as follows :

$$
\begin{aligned}
(X, T) & =\sum_{S \subset N, \mathrm{i} \in \mathrm{s}} \frac{(|S|-1) !(N-\mid S) !}{|N| !}[\mathrm{v}(S)-\mathrm{v}(S-\{\mathrm{i}\})] \\
& =\frac{(|S|-1) !(N-|S|) !}{|N| !} M C,(S) \\
& =\omega(|S|) M C_{\mathrm{i}}
\end{aligned}
$$

$|\mathrm{S}|$ represents the number of participants in the alliance $S$, and $\omega(|S|)$ is the weighting factor of the alliance S. Therefore, the Shapley value can be expressed as the expected value of the marginal contribution of all possible alliance combinations of the participating subject $i$ in the process of cooperative game. The member with more marginal contribution can get more benefits.

The advantage of the above research formula lies in its wide applicability, and the Shapley value can be easily calculated by the formula. Energy Internet should promote joint actions of different participants in energy development strategies under government supervision, pay attention to the distribution of benefits between energy and power grids in various regions, do a good job in the standardization of energy Internet construction, management system and market structure, and ensure that the entire energy Internet is in the background. The interests of the energy alliance are fair, thereby improving the efficiency of energy dispatching and promoting the sustainable development of energy, so as to achieve the goal of building an energy modern society. 


\section{Conclusions and recommendations}

The above is the research and analysis based on the Shapley value in the cooperative game theory in the energy Internet management mechanism. In order to improve energy utilization efficiency, strengthen environmental protection, vigorously develop a green economy, and build a resource-saving and environmentfriendly society, energy Internet management should also focus on the following measures in the future:

1.Lead the overall plan. ${ }^{[6]}$ At present, my country's energy Internet has not yet formed a standard system in various fields, and its foundation can be widely used standards, industry terms, related concepts, framework systems, etc. are not yet complete. The state should mobilize relevant units and authoritative standardization organizations to jointly plan the work of the energy Internet standard system, form a new pattern of crossfield planning and multi-industry joint construction of the Energy Internet Standardization Development Department, and establish as soon as possible the standardization required for the development of the energy Internet in line with China's characteristics The organizational framework is also applied to the project pilot.

2.Use multiple energy to complement each other to improve economic efficiency. ${ }^{[7]}$ Based on the coverage of energy production, transmission and conversion by the Energy Internet, the complementary characteristics of multiple energy sources such as cooling, heating and power are used to tap the time and space complementary potential of various users to improve the economic benefits of the entire integrated energy system.

3.Use precise positioning to provide differentiated services. Grid companies can realize the temporal and spatial interconnection of equipment, users and data, and accurately analyze customer needs and energy consumption information under the background of the energy Internet, so as to facilitate the provision of more accurate and differentiated services in accordance with the energy needs of users.

4.Encourage cross-industry and promote energy technology innovation. Use big data, cloud computing, $5 \mathrm{G}$, artificial intelligence and other technologies to empower the energy Internet, take electric energy as the central link, and use heat, cold, gas and other energy as auxiliary links to establish multi-industry and multisector participation, multi-field coordination, and joint Development of the working mechanism. Explore the establishment of a collaborative mechanism for professional standards organizations such as electricity, cooling, heating, and gas, innovate energy interconnection business models, promote the construction of energy markets, strengthen energy market regulations, and improve the benefit distribution system of the Energy Internet Alliance.

5.Promote international exchanges. Take the initiative to open up, take the initiative to cooperate and exchange with international organizations such as the International Standardization Agency in the field of standard formulation, reflect the role of standards in the construction of the "Belt and Road", promote the concept of energy Internet to the world, and enhance my country's status in the international energy field.

\section{Acknowledgements}

Fund:Research on Social Governance Mechanism Based on Network Game(20191sljdwtkt-027), Liaoning Provincial Federation of Social Sciences 2019.

\section{References}

1. W.J.Shi [J]. China Equipment Engineering, 04,19 8-199(2021)

2. X.B.Yang, Z.F.Tan, H.Y.Lin, F.G.Zhou.J. Aut omation of Electric Power Systems, 689,43 -60(2 020)

3. X.Liu, B.Gao, Z.Zhu, et al. J.IETGeneration, Tra nsmission \& Distribution, 12, 2369-2377.(2018)

4. J.Pu, K.R.Shi, Y.Wang.[J]. Business Economics Research, 000,43-44(2015)

5. C.Y.Lu, T.Jiang, H.Deng, L.Fang, X.Wang, C. W.Jiang.J.Electric Power Construction 483,154-1 62(2020)

6. National Standardization Management Committee and National Energy Administration's Guiding O pinions on Strengthening the Standardization of $\mathrm{E}$ nergy Internet[J]. Power Equipment Management, (2019).

7. Q.D.Dai, X.Deng, X.Y.Wu, Q.Gao, J.A.Zhang, H.W.Wang. [J]. High Voltage Electrical Applia nces, 57,135-144 (2021) 